\title{
A Sticky Situation or Rough Going? Influencing Haptic Perception of Wood Coatings Through Frictional and Topographical Design
}

\author{
Kathryn L. Harris ${ }^{1} \cdot$ Elizabeth S. Collier ${ }^{1} \cdot$ Lisa Skedung $^{1} \cdot$ Mark W. Rutland $^{1,2}$ (D)
}

Received: 8 April 2021 / Accepted: 8 July 2021 / Published online: 6 August 2021

(c) The Author(s) 2021

\begin{abstract}
Improving the tactile aesthetics of products that can be described as touch intensive is an increasing priority within many sectors, including the furniture industry. Understanding which physical characteristics contribute to the haptic experience of a surface, and how, is therefore highly topical. It has earlier been shown that both friction and topography affect tactile perception. Thus, two series of stimuli have been produced using standard coating techniques, with systematic variation in (physical) friction and roughness properties. This was achieved through appropriate selection of matting agents and resins. The stimuli sets were then evaluated perceptually to determine the extent to which discrimination between pairs of surfaces followed the systematic materials variation. In addition to investigating the role of the physical properties in discrimination of the surfaces, their influence on perceived pleasantness and naturalness was also studied. The results indicate that changes in tactile perception can be understood in terms of friction and roughness, and that varying the matting agents (topography) and resins (material properties) in the coatings provide the controlling factors for furniture applications. Perceived pleasantness is associated with low friction and smoother topography, whilst perceived naturalness is found to be described by an interaction between tactile friction and the average maximum peak height of the surface features.
\end{abstract}

\section{Graphic Abstract}

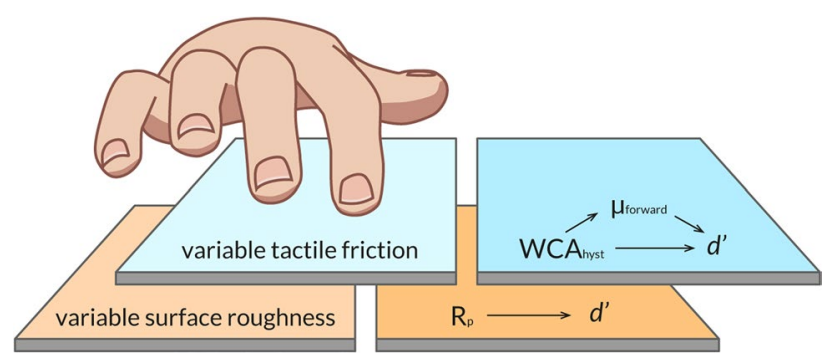

Keywords Haptic perception $\cdot$ Psychotribology $\cdot$ Psychophysics $\cdot$ Tactile friction

\section{Introduction}

Mark W. Rutland

mark@kth.se

1 RISE Research Institutes of Sweden, Malvinas väg 3, 11428 Stockholm, Sweden

2 Department of Chemistry, KTH Royal Institute of Technology, Drottning Kristinas Väg 51, 11428 Stockholm, Sweden
Understanding which factors contribute to the haptic experience of a surface is important; none of our other senses interact so persistently with our surroundings. The increasing prevalence of touch intensive consumer products such as mobile phones and tablets has increased general awareness of the science of touch but represents just a fraction of the contribution of touch in quality assessment. Haptic evaluation of objects before purchase can influence the decision made [1]. Improving the tactile aesthetics of products 
that frequently come into contact with the skin is thus an increasing priority within many industries [2], including the furniture industry. Although crucial to the implementation of a quality control protocol based on haptic properties, the effects of changes to physical parameters that contribute to the haptic perception of surfaces for furniture solutions are not yet fully understood.

When a surface is touched, a complex tribological interaction occurs between the finger and that surface. The resultant haptic experience is affected by deformation of the skin due to pressure and lateral movement $[3,4]$, the topography of the surface [5], the frictional forces [6] as well as mechanical vibrations induced by sliding the finger [7-10]. The human finger is sensitive to texture differences at the nanoscale [6] and to differences between nearly perfectly smooth surfaces that differ only in their chemical composition $[11,12]$. In short, it is exquisitely sensitive to even very small differences in the properties of surfaces. It is thus also clear that tribology, or Psychotribology [12], plays an important role in the understanding of tactile perception as a whole.

It has been suggested [13] that there may be up to five dimensions of haptic touch; three fundamental dimensions (rough/smooth, hard/soft and cold/warm) where the rough/ smooth dimension is further subdivided into fine (micro) and coarse (macro) textures, and friction (moist/dry or sticky/ slippery) is the fifth dimension. Determining the physical parameters of surfaces that are related to these dimensions of haptic touch has garnered a great deal of attention across the past decades. Some recent work has even focussed on 3D-printed stimuli to precisely vary surface statistics [14]. Using a large set of 124 different stimuli, Bergmann Tiest and Kappers [15] assessed the relationships between physical measurements of compressibility and roughness and participants' perceptions of these attributes, whilst Skedung and colleagues $[6,16]$ have investigated the roles of wavelength spacing and friction in facilitating discrimination of periodically patterned stimuli, and demonstrated how these factors were important in people's ability to distinguish between fine textures. Additionally, it has been found that differences in the perceptions of slippery-sticky, wet-dry, and hard-soft appeared to be related to variations in multiple physical aspects [17]. For example, sticky-slippery was related to differences in the cooling rate, compliance, tactile friction coefficient, and a general representation of surface roughness (in this case the average of the surface peaks and valleys from the mean line, $R_{\mathrm{a}}$ ). This illustrates the complexity of modelling haptic perception against variation in physical parameters, as several physical characteristics can nearly always be related to a single haptic dimension.

Surface topography is a salient aspect of haptic perception, often of primary importance. Many surface features can affect roughness perception, for instance wider spacing and narrower features can increase perceived roughness [18].
Although physical roughness and roughness perception have been investigated, it has been pointed out that there appears to be a lack of work assessing the relative contributions of different aspects of roughness [19]. In general, the focus is on single height parameters such as $R_{\mathrm{a}}$, which by no means uniquely define the surface topography. Although such parameters may be appropriate for general characterisation within more narrow contexts, a more exhaustive description is needed in order to describe the complexity of a surface in the context of haptic perception [19]. Additional factors such as feature shape and spacing are relevant since, via contact mechanical considerations, the morphology of the surface features affect the interaction between the surface and the finger, which in turn impacts the tactile experience [20,21].

In this study, two series of stimuli were produced using standard furniture coating techniques in order to more systematically investigate how variation in friction and different roughness parameters influences haptic perception-in this case of wood coatings. One series of samples was developed to vary predominantly in its frictional character (sticky/slippery) through the use of different resins (all of which were polyester or polyether acrylic oligomers) in the topcoats, whilst the second was developed to vary in surface texture by including different silica- or polyurethanebased matting agents in the topcoats. In addition to measuring the tactile friction and a range of physical roughness parameters, water contact angle (WCA) measurements were included in an attempt to more fully describe the aspects of perception that may not be revealed via friction and roughness evaluations alone. Previous work has indicated a relationship between the WCA (separately from the tactile friction coefficient) and the perception of smooth surfaces varying only in the chemistry of a molecularly thin coating [12] so this technique could also be relevant for capturing surface energy effects in this case. It should, however, be noted that the influence of roughness on the measured WCA cannot be ignored [22].

In addition to investigating the role of physical properties in haptic discrimination of the surfaces, other aspects of the haptic experience of the surfaces were also assessed. Of particular interest in this work was the effect on the perception of naturalness and pleasantness by touch. Labbe et al. [23] found that of the three modalities they tested (touch, vision and audition), touch was the primary contributor to perceived naturalness across a range of materials including fabrics and papers, and it has been previously shown that friction is negatively associated with perceived pleasantness $[12,24]$. People tend to prefer natural materials over synthetic ones [25] and so it is thus a priori reasonable to expect that people should prefer materials they perceive as more natural, regardless of the actual source of the material. Overvliet and Soto-Faraco [25] showed that there was a good degree of consistency between assessments of naturalness 
of their stimuli using different psychophysical methods, suggesting the existence of some underlying construct of naturalness. It is thus to be expected that furniture consumers would prefer products that feel more natural. However, retaining a natural feeling whilst simultaneously improving the cleanability and durability of furniture through the addition of coatings is challenging. Measuring perceived naturalness of the two sets of stimuli used in this experiment is one of the first steps towards describing which of the physical parameters that can be controlled during the manufacturing process of furniture coatings contribute most to a perception of naturalness by touch alone.

In summary, the aim of the present work was to attempt to manipulate haptic perception of two sets of surfaces by systematically varying their friction or roughness characteristics. These parameters cannot be entirely deconvoluted, since there is also second-order variation in the "other" parameter. It was nonetheless hypothesised that the relative importance of the variation in friction or roughness would influence perception to different degrees in each set. Further, perceived pleasantness and naturalness, as well as other perceptual attributes of the surfaces, were measured and how variation in physical parameters affected these attributes was assessed.

\section{Methods}

The experiments involving humans presented in this manuscript were conducted in accordance with the Declaration of Helsinki (2013). When collecting and processing personal data, the principles of the General Data Protection Regulations (GDPR) were adhered to. Informed consent was obtained in writing from all participants before the experiment began.

\subsection{Stimuli}

The coatings were applied using a roller system to MDF sheets and all included a clear base layer of filler, a sealant, three layers of white pigmented base coat and a topcoat. Each layer was UV cured (Hg lamp) before the next was applied, and the sealant layers were industrially machine sanded with 320 and 400 grit sandpaper before the succeeding steps. The compositions of the UV topcoats were varied systematically to create two series of stimuli, henceforth referred to as Series 1 and 2, each containing six samples. Each set included a 'standard' coating, formulated with the rest of the members of the series in mind. All coatings were applied in layers between 5 and $25 \mathrm{~g} / \mathrm{m}^{2}$, depending on the desired result.

In Series 1, the resins in the topcoats were varied. This was hypothesised to generate variations in the perceived hardness, softness and stickiness of the samples' surfaces which in turn were thought to affect the frictional interface. (Variation in the elastic modulus of polymers of identical roughness has been shown to be detectable both via perception pathways and by the friction coefficient. The combined contact mechanical deformation of the stratum corneum and the polymer itself are responsible for these differences) [21]. All of the topcoats in this series included $3 \%$ silica particulate matting agent (mean diameter $8 \mu \mathrm{m}$ ) by mass. The standard in Series $1(1 \mathrm{~A})$ was a polyester acrylate oligomer topcoat. Five other coatings were created using three other resins in varying proportion to the standard. The topcoats on samples $1 \mathrm{~B}$ and $1 \mathrm{D}$ were based

Table 1 Shows the matting agent, resin, mean ( \pm standard deviation) of the measured $R \mathrm{p}$, coefficient of tactile friction $(\mu)$ and water contact angle hysteresis for each of the six samples in each of Series 1 and 2

\begin{tabular}{|c|c|c|c|c|c|c|}
\hline Series & Sample & Matting agent & Resin & $R_{\mathrm{p}}(\mu \mathrm{m})$ & $\mu$ & $\mathrm{WCA}_{\text {hyst }}\left({ }^{\circ}\right)$ \\
\hline 1 & $1 \mathrm{~A}$ & Silica particulate (3 wt \%) & Polyester acrylate oligomer & $3.8 \pm 1.5$ & $1.0 \pm 0.3$ & $45 \pm 13$ \\
\hline 1 & $1 \mathrm{~B}$ & Silica particulate (3 wt \%) & $\begin{array}{l}\text { Flexible aliphatic urethane acrylate oligomer: } \\
\text { composition } 1\end{array}$ & $0.6 \pm 0.1$ & $1.9 \pm 0.5$ & $59 \pm 6$ \\
\hline 1 & $1 \mathrm{C}$ & Silica particulate (3 wt \%) & $50: 50$ mixture of $1 \mathrm{~A}$ and $1 \mathrm{~B}$ & $1.2 \pm 0.3$ & $1.6 \pm 0.3$ & $58 \pm 5$ \\
\hline 1 & $1 \mathrm{D}$ & Silica particulate (3 wt \%) & $\begin{array}{l}\text { Flexible aliphatic urethane acrylate oligomer: } \\
\text { composition } 2\end{array}$ & $1.1 \pm 0.9$ & $2.2 \pm 0.5$ & $62 \pm 24$ \\
\hline 1 & $1 \mathrm{E}$ & Silica particulate (3 wt \%) & $50: 50$ mixture of $1 \mathrm{~A}$ and $1 \mathrm{~F}$ & $2.8 \pm 0.4$ & $0.9 \pm 0.3$ & $46 \pm 2$ \\
\hline 1 & $1 \mathrm{~F}$ & Silica particulate (3 wt \%) & Highly functionalised aliphatic urethane oligomer & $2.6 \pm 0.3$ & $1.0 \pm 0.5$ & $47 \pm 13$ \\
\hline 2 & $2 \mathrm{~A}$ & Silica particulate (12 wt \%) & Polyester acrylate oligomer & $5.1 \pm 0.5$ & $0.9 \pm 0.1$ & $53 \pm 7$ \\
\hline 2 & $2 \mathrm{~B}$ & Polyethylene dispersion wax (13 wt \%) & Polyester acrylate oligomer & $2.1 \pm 0.2$ & $1.6 \pm 0.4$ & $52 \pm 9$ \\
\hline 2 & $2 \mathrm{C}$ & Crosslinked polyacrylic ester (14 wt \%) & Polyester acrylate oligomer & $6.2 \pm 0.7$ & $0.7 \pm 0.1$ & $53 \pm 14$ \\
\hline 2 & $2 \mathrm{D}$ & Polyurethane $(14 \mathrm{wt} \% ; \mathrm{MPD}=30 \mu \mathrm{m})$ & Polyester acrylate oligomer & $9.3 \pm 0.7$ & $0.8 \pm 0.1$ & $52 \pm 20$ \\
\hline 2 & $2 \mathrm{E}$ & Polyurethane $(12$ wt $\%$; MPD $=7 \mu \mathrm{m})$ & Polyester acrylate oligomer & $4.2 \pm 0.2$ & $0.9 \pm 0.2$ & $53 \pm 3$ \\
\hline 2 & $2 \mathrm{~F}$ & $\begin{array}{l}\text { Polyurethane matting agent ( } 13 \mathrm{wt} \% \text {; } \\
\mathrm{MPD}=8 \mu \mathrm{m})\end{array}$ & Polyester acrylate oligomer & $5.5 \pm 1.1$ & $0.8 \pm 0.1$ & $49 \pm 18$ \\
\hline
\end{tabular}


on the same flexible aliphatic urethane acrylate oligomer of different compositions: in both cases the resin compositions were designed for a lower elastic modulus. Sample $1 \mathrm{C}$ was coated with a 50:50 (by mass) mixture of the $1 \mathrm{~A}$ and $1 \mathrm{~B}$ formulations. Topcoat $1 \mathrm{~F}$ was a highly functionalised aliphatic urethane oligomer with a high number of crosslinks leading to a harder cured film. Sample 1E was a 50:50 (by mass) mixture of the $1 \mathrm{~A}$ formulation and topcoat $1 \mathrm{~F}$.

In the second series (Series 2), various different matting agents were used which was hypothesised to generate variations in the perceived textural qualities of the samples. The standard for Series $2(2 \mathrm{~A})$ used the same silica matting agent as sample $1 \mathrm{~A}$, but in higher concentration (12 wt \%). Sample 2B's topcoat was based on a polyethylene dispersion wax (13 wt \%). The matting agent in sample 2C was a crosslinked polyacrylic ester $(14 \mathrm{wt} \%)$ with mean particle diameter $15 \mu \mathrm{m}$. Samples 2D, 2E and 2F all had polyurethane matting agents $(14,12$ and $13 \mathrm{wt} \%$, respectively), with mean particle diameters (MPD) 30,7 , and $8 \mu \mathrm{m}$, respectively. Table 1 summarises the matting agent, resin, tactile friction coefficient, maximum peak heights of the profile elements $\left(R_{\mathrm{p}}\right)$ and water contact angle hysteresis of each surface within each set.

\subsection{Physical Characterisation}

\subsubsection{Tactile Friction}

Tactile friction was measured with a ForceBoard ${ }^{\mathrm{TM}}$ (Industrial Dynamics AB, Sweden), a universal friction and force tester equipped with one horizontal and one tangential load cell. A mechanical load is converted into voltage signals which are amplified and proportional to the applied load. The tangential force, i.e. friction force, and vertical force, i.e. applied load, were recorded using DAQFactory software at a sampling rate of $1000 \mathrm{~Hz}$ whilst a finger was moved regularly over the samples, which were rigidly attached to the plate. The output data were further analysed in MATLAB using custom code.

Samples were wiped clean of dust and debris using ethanol and laboratory wipes. The friction measurements were conducted by a single female operator (age 28 years), with the finger held at approximately $30^{\circ}$ to the surface, using a by now well-documented technique [6, 16, 21]. An applied load of $1 \mathrm{~N}$ was maintained to the best ability of the operator (mean: $0.97 \mathrm{~N}, \mathrm{SD}: 0.04 \mathrm{~N}$ across all tests, the operator is able to monitor the load whilst sliding), and the sliding velocity was approximately $75 \mathrm{~mm} / \mathrm{s}$. The operator completed seven measurements for each stimulus type. Each measurement consisted of ten reciprocations and was performed at a new location on the surface. Dynamic friction coefficients were calculated as the instantaneous ratio of friction force to applied load. The acquired data in the central $40 \%$ (calculated about the centre of each stroke as defined by the temporal midpoint between the $0 \mathrm{~N}$ lateral load data regions resulting from the change of sliding direction) of each cycle were used for all calculations in order to eliminate "turn around" effects. Note that the friction coefficient generated by this approach provides a physical characterisation of the surface-it should not be seen as exactly describing the friction experienced by participants as they probe surfaces-that will depend on the load, speed, and moisture levels of the individual fingers.

\subsubsection{Roughness}

Using a Dektak XT stylus profilometer with a stylus tip of $2 \mu \mathrm{m}$ radius, $50-\mathrm{mm}$-long line scans were performed in three locations on each of three examples (nine measurements total) of each coating at a resolution of $3 \mu \mathrm{m} / \mathrm{pt}$. All scans were tilt and curvature corrected. Line scans were processed with a gaussian regression filter using a standard $25-\mu \mathrm{m}$-short wavelength cutoff, a 0.8 -mm-long wavelength cutoff and a $1 \%$ sampling length as in [19]. These cutoff wavelengths were chosen based on stylus tip radius, visual inspection of the roughness profiles as compared to the primary profile and the successful use of a 0.8 -mm-long wavelength cutoff in other studies investigating perception [26]. Roughness/surface parameters were extracted using Vision 64 Software.

\subsubsection{Water Contact Angle}

Static, advancing and receding water contact angles were measured with a Dataphysics OCA-40 micro instrument using ultrapure water (Merck MilliQ Integral). Static measurements were taken using a $5 \mu \mathrm{L}$ drop after approximately $10 \mathrm{~s}$ of equilibration. Advancing and receding measurements were performed at a dispensing rate of $0.5 \mu \mathrm{L} / \mathrm{s}$. Values presented are the average of angles taken from the left and right edges of three drops on each of three examples of each surface. Roughness corrections to the contact angles were not performed, the implications of which are discussed where relevant.

\subsection{Perception Tests}

Ten participants (mean age $=25.2$ years, $\mathrm{SD}=4.05$ years; 4 female) were recruited using an online recruitment service. The participants gave their informed consent before beginning the experiment and received a gift certificate as compensation for their time.

After signing the consent form and washing their hands with liquid hand soap and water, participants sat at a table in front of a large screen with a hole covered by a curtain, 

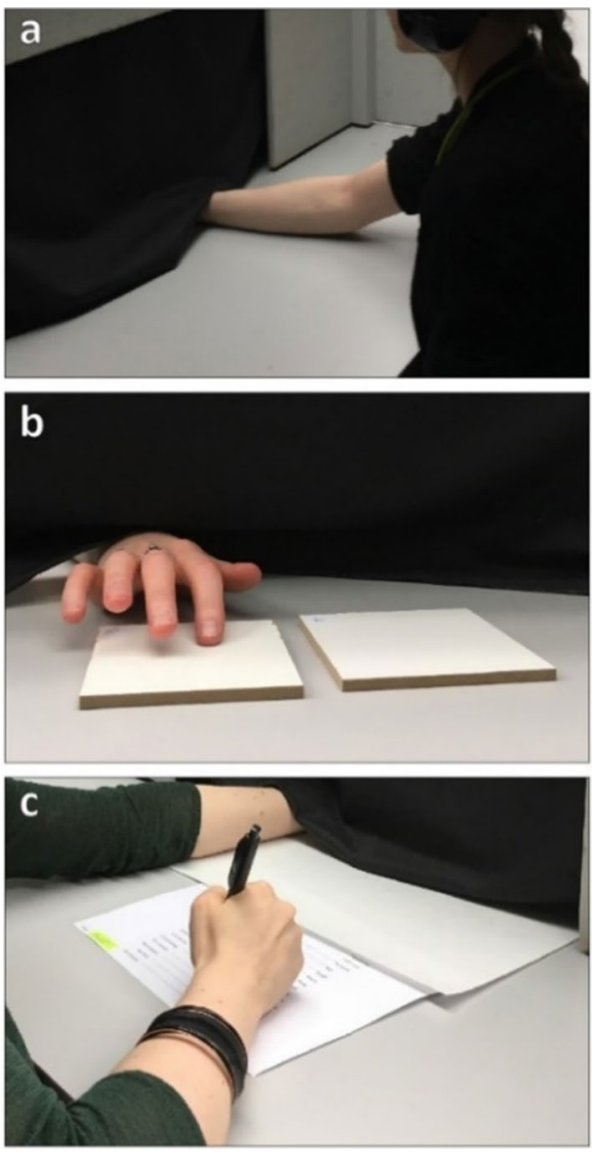

Fig. 1 Setup and procedure of the same-different task (a, b) and adjective ratings task (c)

see Fig. 1a. The participants were presented sequentially with two stimuli on each trial and their task was to respond whether the stimuli felt the same or different. The participants could touch each sample for as long as they liked, and however they liked, but only using their non-dominant index finger. The task was completed for Series 1 and Series 2 in two separate blocks of trials performed on the same occasion. Within each block, every stimulus was compared to every other stimulus in that series, including itself, and each pair was repeated twice in each possible order, for a total of 72 trials in each block. There was a short break between the two blocks. Half of the participants completed the Series 1 block first followed by the Series 2 block, and vice versa for the other half of participants. The presentation order within each block was randomised uniquely for each participant.

From the participants' responses in the same-different task, the proportion of hits $[p$ (response $=$ "Different"|Diff erent)] and false alarms [ $p$ (response $=$ "Different"'Same)] were calculated for each participant, and the sensitivity parameter $d$ ', based on the differencing method [27], was then estimated. Although the hit rate by itself could be used as a measure of participant's accuracy, this would mean that

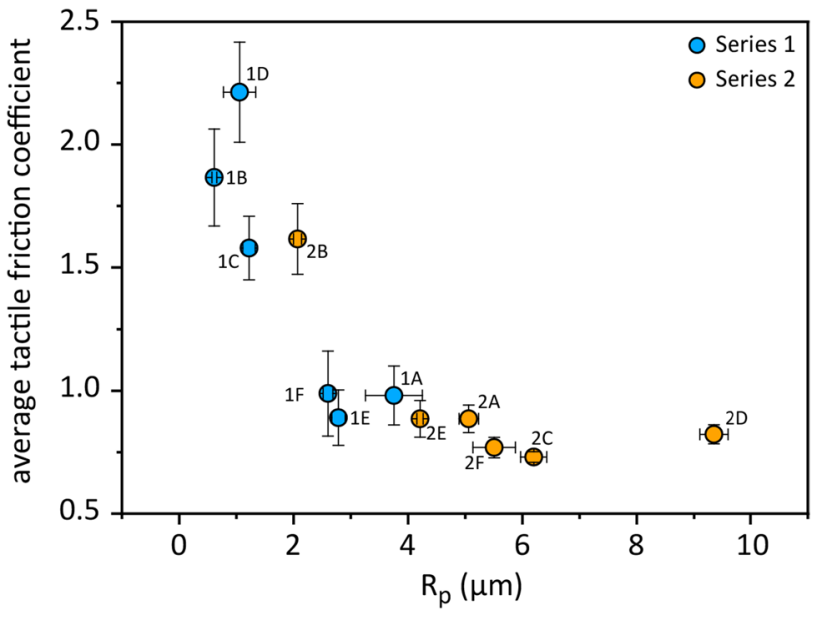

Fig. 2 The spread of the stimuli in Series 1 and 2 by $R \mathrm{p}$ and average tactile friction coefficient. Error bars represent the standard errors of the means

the occasions where they guessed would be weighted the same as when they were correct. The incorporation of the false alarm rate in the calculation of $d$ ' means that a better picture of participant accuracy can be gleaned, as it is then possible to account for their ability to detect when samples are actually different as well as when they incorrectly identify the same samples as different. Thus, $d$ ' is commonly used in psychophysics as it is a more comprehensive description of the participants' discrimination ability than the hit rate or \% correct alone [27]. However, in the present study, there were many instances where either the hit rates or false alarms were 0 or 1 . Since $d$ ' cannot be determined when hit rates and/or false alarms are 1 or 0 [27], typically values of 0 are corrected to the value $[2 \mathrm{~N}]^{-1}$ and values of 1 are corrected to be equal to $\left[1-(2 \mathrm{~N})^{-1}\right]$. Here, $\mathrm{N}$ is the maximum number of trials on which a false alarm or hit could have occurred. To minimise the number of corrections necessary to be able to calculate $d$ ', instead collapsed $d$ ' was calculated across all participants for each pair of stimuli [28].

Following this, a second task was performed. The participants felt the stimuli one at a time with their non-dominant index finger whilst rating the surface on a set of visual analogue scales (VAS; shown in the ESI) to reflect how they perceived it. These were 20 adjective scales based on pairs of opposing terms separated by a $10-\mathrm{cm}$-long horizontal line [ESI Fig. S1]. The adjective pairs were appealing-unappealing, velvety-not velvety, coarse-fine, rough-not rough, warm-cold, cheesy-not cheesy, hard-not hard, desirable-undesirable, sticky-not sticky, smooth-not smooth, wet-dry, mainstream-not mainstream, expensive-cheap, soft-not soft, high quality-low quality, interesting-boring, slippery-not slippery and, crucially, pleasant-unpleasant and natural-synthetic. The ratings task was primarily to 
facilitate interpretation of the outcome of the same-different task by highlighting which perceptual dimensions the stimuli might perceived to be different in. The samples were presented in a unique random order for each participant and the adjective order for each sample was also randomised. In both tasks, the stimuli were presented behind the curtain, and the participant placed their non-dominant hand through the curtain to touch them (Fig. 1b). All participants completed both tasks for both sets of stimuli.

\section{Results}

\subsection{Physical Characterisation}

The two series of stimuli were primarily produced in an attempt to more systematically investigate how variation in the friction character (Series 1, "sticky/slippery") and surface texture (Series 2, "rough/smooth") can influence haptic perception of wood coatings. To demonstrate the respective variation in these variables, every surface in the two series is plotted in Fig. 2 in terms of the friction coefficient and the average maximum peak heights of the roughness profile $\left(R_{\mathrm{p}}\right)$. It can be seen that Series 1 varied mainly in terms of the tactile friction coefficient, whilst Series 2 showed a significant spread in roughness and less variation in tactile friction coefficient. As discussed above, these two properties are generally related and roughness, especially in the scale on which these coatings fall $(0-10 \mu \mathrm{m})$, is difficult to vary entirely independently of the friction coefficient when the materials are held constant [29].

One-way ANOVAs revealed that both Series 1 and 2 varied significantly in mean friction coefficient (both $p<0.001$ ); in Series 2 this can mainly be attributed to the higher friction of (smoother) sample 2B relative to the other five, see Fig. 2. Both series also varied significantly in $R_{\mathrm{p}}$ (both $p<0.001$ ). This is expected: roughness and friction are intimately related. Nonetheless, the control of matting agents in Series 2 successfully led to systematic topographical variation over a large range. Similarly, the use of different resins, where the elastic modulus is changed, led to the desired variation in friction properties in Series 1, over a range where it is known that there is significant perceptual discrimination [6, 30]. Although the differences in $R_{\mathrm{p}}$ of the samples in Series 1 (friction) are in fact statistically significant, the range of variation is nonetheless considerably smaller $(\sim 0.5-4 \mu \mathrm{m})$ than that in Series $2(\sim 1-10 \mu \mathrm{m})$. Conversely, though the friction coefficient of the samples in Series 2 is necessarily somewhat affected by the roughness value (e.g. 2B), the friction variation is much greater in Series 1 . The level of covariation was deemed sufficiently low that it should not preclude tactile discrimination experiments in the series. This hypothesis is tested directly in the sections that follow.
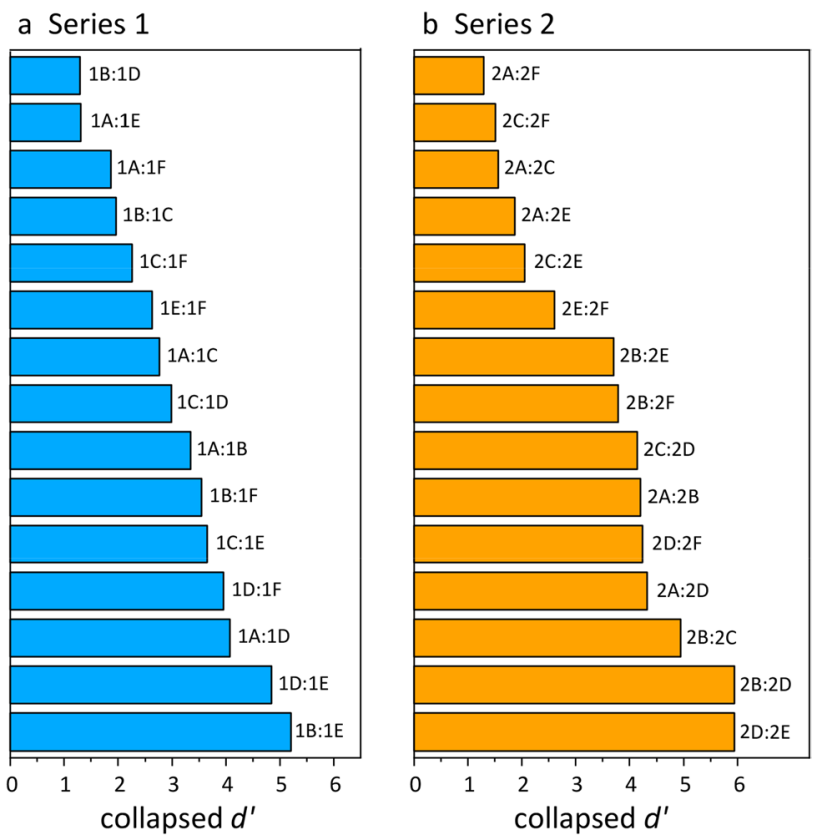

Fig. 3 The collapsed $d$ ' values for each of the comparisons presented to participants (Series 1 in $\mathbf{a}$, Series 2 in $\mathbf{b}$ ). No error bars were generated for collapsed d', because it represents performance collapsed across all the participants. Bar labels indicate the surface pair in question

Table 2 Fisher's $z$ tests assessing the relative strength of the correlations (Pearson's $r$ ) between $d$ ' and differences in the mean coefficient of friction and $R_{\mathrm{p}}$ across Series 1 and 2

\begin{tabular}{lllll}
\hline & $r\left(d^{\prime}\right.$ Series 1) & $r\left(d^{\prime}\right.$ Series 2$)$ & Fisher's $z$ & $p$ (one-tailed) \\
\hline $\begin{array}{l}\text { Friction } \\
\text { coeffi- } \\
\text { cient }(\mu)\end{array}$ & 0.86 & 0.46 & 1.92 & $0.027^{*}$ \\
$R_{\mathrm{p}}(\mu \mathrm{m})$ & 0.54 & 0.94 & -2.74 & $0.003^{* *}$ \\
\hline
\end{tabular}

One-tailed $p$ values are reported because the hypotheses were directional in both cases

$* p<0.05$

$* * p<0.01$

\subsection{Perception Tests and Describing d' with Physical Parameters}

\subsubsection{Discriminability $\left(d^{\prime}\right)$}

Discriminability between pairs was calculated as described in the methods (perception tests) section. Figure 3a, b shows that the discriminability varied widely across the different pairs within each series. A large value of collapsed $d$ ' indicates that the pairs were easily discriminated, a low value means that the task was more difficult.

In Series 1, the surfaces in the most discriminable pair (1B:1E) were always identified as different by all 
participants, and had a false alarm (responding "different" on trials when a sample is compared to itself) rate of 0.15 , whilst the least discriminable pair (1B:1D) had participants correctly identify the pair as "different" with a rate of 0.2 , with a false alarm rate of 0.075 . In Series 2, participant performance for the most discriminable pairs (2B:2D and 2D:2E) was nearly perfect for all participants, whilst the least discriminable pair (2A:2F) had a hit rate of 0.275 and a false alarm rate of 0.125 .

To directly test the success of the haptic design hypothesis (i.e. that the samples in the two series are predominantly discriminable by "friction" and "roughness"), Fisher's $r-z$ transformation was performed. The Fisher's $z$ test is a means of testing whether the strength of different correlation coefficients differ significantly to each other rather than whether they are significant in themselves. In other words, it facilitates comparison of the relative strength of two correlation coefficients; in this case the correlation coefficients between $d^{\prime}$ and $R_{\mathrm{p}}$, and between $d^{\prime}$ and the friction coefficient across Series 1 and 2. It was expected that the correlation between $d$ ' and the difference in friction would be significantly stronger in Series 1 than Series 2, and vice versa for $R_{\mathrm{p}}$. Both hypotheses were supported by the analyses, see Table 2 , indicating that although both series varied in both parameters as measured instrumentally, the parameters were relevant for human discrimination to different degrees across the two series.

The results of multivariate analysis of variance (MANOVA) on the adjective ratings for each of Series 1 and 2 can be found in the ESI. Correlation analysis was performed between $d$ ' and the mean absolute differences $\left[\operatorname{ABS}\left(\bar{X}_{\text {sample1 }}-\bar{X}_{\text {sample2 }}\right)\right]$ of adjective ratings between pairs of samples within each series as a way of gauging which physical characteristics may be relevant for discrimination of the different pairs.

\subsubsection{Series 1}

Within Series 1, there was a significant correlation between $d$ ' and differences between samples in ratings of stickiness $[r=0.74, p=0.002]$. No other significant correlations between $d$ ' and adjective ratings were found. The physical parameters whose differences between samples strongly correlated with $d$ ' were the water contact angle hysteresis, $[r=0.80, p<0.001]$, the receding water contact angle $[r=0.78, p<0.001]$, and all three measures of friction coefficient that were calculated: the overall mean, $[r=0.86, p<0.001]$, the mean in the reverse direction $[r=0.82, p<0.001]$ and the mean in the forward direction $[r=0.88, p<0.001]$.

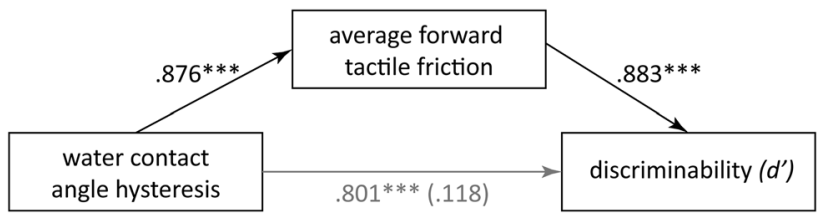

Fig. 4 Shows the mediated relationship between WCA hysteresis and $d^{\prime}$. The values are standardised regression coefficients and the stars show statistical significance $(* * * p<.001)$. The value in parentheses is the standardised regression coefficient between WCA hysteresis and $d^{\prime}$ ' when average forward friction is included in the same model. The mediation was statistically significant $(p<0.001,95 \%$ confidence interval $=0.05-0.27$ ) and suggests that $d^{\prime}$ was related to WCA hysteresis because of the related changes in forward friction experienced during interaction, rather than being directly impacted by the WCA hysteresis

Because of the nature of the variation within Series 1 (chemical differences in the resins), the strong relationships of $d$ ' with the friction coefficients and the contact angles were presumed to be related to one another rather than complementary. We therefore used the Mediation package in $\mathrm{R}$ (version 3.6.1, The R Foundation for Statistical Computing Platform) to test whether the forward tactile friction (the strongest correlation with $d$ ') mediated the relationship between WCA hysteresis and $d$ '. Mediation occurs when the variance explained by one factor is shown to be due to another related factor. In other words, mediation analysis allows us to describe how a factor affects a response, in this case how differences in the WCA hysteresis affect tactile perception. Using bootstrapping procedures based on 500 simulations on random subsamples from the data, it was found that the effect of differences in the WCA hysteresis on $d$ ' was mediated by differences in the forward tactile friction coefficient $(p<0.001 ; 95 \%$ confidence interval $=0.05-0.27)$. This mediated relationship also exists (to a different degree) for the overall average friction coefficient, but not for the reverse friction coefficient. This analysis indicates that $d$, was related to differences in the WCA hysteresis, but this can be explained in terms of the resultant variations in tactile friction, which provided the cue that our participants likely perceived (see Fig. 4).

\subsubsection{Series 2}

Within Series 2, there were significant correlations between $d^{\prime}$ and differences in ratings of roughness $[r=0.59, p=0.021]$, slipperiness $[r=0.85, p<0.001]$, smoothness $[r=0.70, p=0.004]$, softness $[r=0.77$, $p=0.76, p<0.001]$, pleasantness $[r=0.62, p=0.013]$ and wetness $[r=0.69, p=0.004]$. Further correlation analysis showed that perceived smoothness, slipperiness 
and softness correlated with the maximum profile peak height, $R_{\mathrm{p}}[r=-0.97, p=0.002 ; r=-0.89, p=0.017$; and $r=-0.96, p=0.003$, respectively], and that perceived stickiness and wetness correlated with the mean tactile friction coefficient $[r=0.87, p=0.024$ and $r=0.89$, $p=0.019$, respectively $]$.

The roughness parameter $R_{\mathrm{p}}[\beta=0.77]$ was identified as a significant contributor to the linear model for $d$ ', accounting for around $88 \%$ of the variance in the data $\left[\mathrm{R}^{2}=0.88, F(1,13)=95.60, p<0.001\right]$, suggesting that discrimination in Series 2 was almost entirely related to absolute differences between feature heights on the surfaces of the samples. Many of the other measured roughness parameters correlated well with $d^{\prime}$, but the fit with $R_{\mathrm{p}}$ was the strongest. It is reasonable from a contact mechanical perspective that topographical peaks contribute significantly to the interaction during haptic touch whilst the depth and shape of valleys analogously becomes less relevant. The simplified topographical description provided by $R_{\mathrm{p}}$ thus appears to provide a sufficient framework to explain the observed discrimination between these surfaces. The results indicate that greater differences in $R_{\mathrm{p}}$ between a pair of samples are associated with easier discrimination in active touch for Series 2.

\subsubsection{Perceived Pleasantness and Naturalness}

In addition to the ability to control haptic perception of surfaces in terms of their discriminability from other, similar, surfaces, the resulting subjective evaluation of specific coating constituents is also of great perceptual relevance. As discussed in the introduction it is expected that both friction and roughness would contribute to perceptions of naturalness and pleasantness, so an analysis of how variations in the tactile friction and $R_{\mathrm{p}}$ of the coatings across both series affect them, is now undertaken. Instead of analysing the two series separately, here the data were analysed together. This is because the ratings task was not run separately for each series and, moreover, to adequately model variance in attributes such as pleasantness and naturalness more variety in the samples is advantageous.

A significant model $\left[\mathrm{R}^{2}=0.78, F(3,8)=9.17, p=0.006\right]$ for perceived naturalness included tactile friction $[\beta=14.57$, $p=0.2]$ and $R_{\mathrm{p}}[\beta=20.53, p 0.013]$ as factors, including an interaction term $[\beta=-20.71, p=0.03]$ as illustrated in Fig. 5 (left). This interaction indicates that for surfaces with higher average maximum peak height, increases in the friction coefficient are associated with a steep decline in perceived naturalness, whereas for surfaces with lower $R_{\mathrm{p}}$, the influence of friction is far less, and these surfaces are expected to be perceived as less natural in general. The two series included low roughness/high friction samples, and high roughness/low friction samples, but no high friction/ high roughness samples, since such samples are very difficult to achieve without significant variation in the surface chemistry or additive geometry. Thus, the predicted ratings of low pleasantness and low naturalness in that section of the plot are projections based on the model and should consequently be treated with caution. (For a sample to have a high friction coefficient at a higher roughness level, the surface must either be quite adhesive/of very low modulus, or the roughness large or sharp enough, to instigate a ploughing mechanism in the friction-or both.) It should also be noted that the surfaces used in this experiment can hardly be considered natural, and yet a basic model for how their physical characteristics are related to their respective, albeit low, ratings of naturalness can nonetheless be identified. The

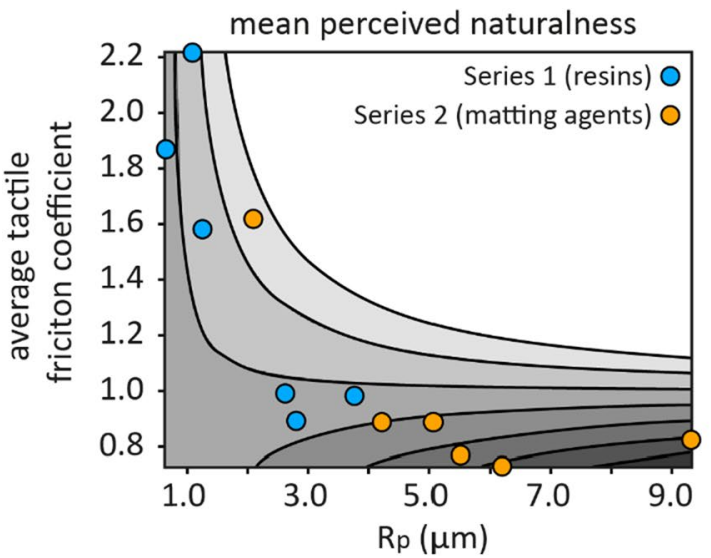

Fig. 5 Contour plots illustrating the predicted effects of variations in friction and roughness on the perceived pleasantness and naturalness of the samples in Series 1 (red markers) and 2 (yellow markers). Left: Shows the interacting effects of friction and roughness on the per-

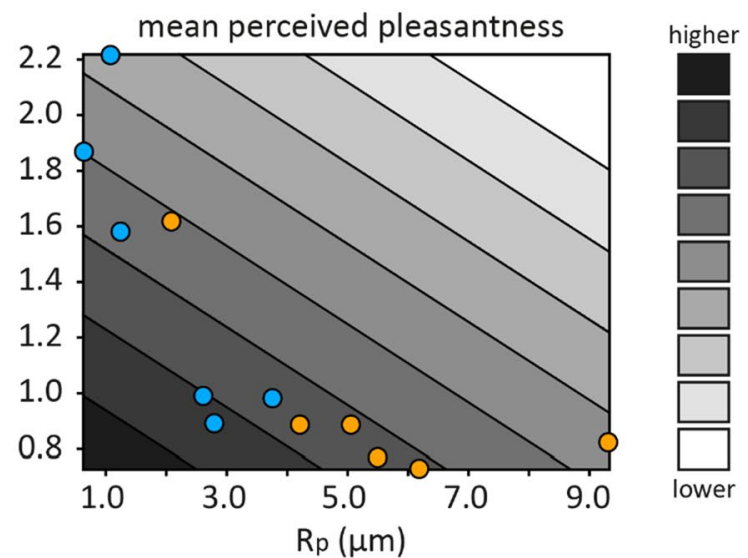

ceived naturalness of the samples. The relationship is non-monotonic, but the most natural surfaces are expected to be low friction and high roughness. Right: Indicates that perceived pleasantness is expected to be higher for surfaces with low friction and low roughness 
analyses suggest that perceived naturalness by touch seems to be associated with rougher surfaces.

For perceived pleasantness, a significant model with $R_{\mathrm{p}}$ $[\beta=-4.84, p=0.008]$ and friction $[\beta=-34.53, p=0.039]$ as factors was found $\left[\mathrm{R}^{2}=0.56, F(2,9)=5.71, p=0.025\right]$, see Fig. 5 (right). This analysis suggests that surfaces with high roughness and high friction would be less preferred, whilst low friction, low roughness surfaces were the most preferable to touch. Caution is again urged when considering the pleasantness values predicted for high friction/high roughness samples, as none of the samples tested here were in this range.

\section{Discussion}

The aim of this experiment was to test the extent to which intentional manipulation of the friction character and surface roughness of furniture coatings could systematically influence haptic perception via changes in the matting agents and resins. As hypothesised, although Series 1 and 2 each varied significantly in both roughness and friction, they did so to markedly different degrees and, importantly, these two factors were relevant for discrimination within each series to correspondingly very different extents.

Interestingly, for Series 1, although there was a strong relationship between the water contact angle hysteresis and $d$ ', the forward friction coefficient relationship appeared to mediate this effect, meaning that much of the variation explaining differences in discriminability of the surface pairs thought to be due to the WCA hysteresis was in fact due to the resultant variation in the forward friction generated by the WCA hysteresis, which itself describes the activation energy of changing the contact area between the water droplet and the surface [31]. The friction coefficient in the forward direction is thus thought to be the primary cue relating to $d$ ' here, suggesting that changes to the friction coefficient (by way of modifications to the resins in the coatings), particularly that experienced during the motion of the finger away from the body, were strongly related to participants' ability to distinguish between the surfaces.

The improvement of the fit for the forward friction coefficient as compared to the average or reverse values may represent the effect of mechanical asymmetry of the finger and its effect especially at higher values of the friction coefficient, where the finger is more likely to be caused to bend at the joints. This mechanical asymmetry also leads to differences in the contact area, both due to the curling of the finger in the forward (distal) direction and the pushing together of finger ridges in the reverse (proximal) direction [32]. This effect has the result of reducing the contact area, and thus increasing the contact pressure [4], in the forward direction (the load was held quite constant throughout testing; avg. forward $=0.998 \mathrm{~N}, \mathrm{sd}=0.08 \mathrm{~N}$, avg. reverse $=0.948 \mathrm{~N}$, $\mathrm{sd}=0.05 \mathrm{~N}$ ). This likely led to the slightly higher degree of variation in the friction coefficient in the forward direction than in the reverse. The amplification of the effects of high friction on the finger in the forward direction is thus a likely reason that $d$ ' was best described by the forward friction coefficient, though the average and reverse values did not represent a large change to the correlation coefficient. (A recent model suggests that in the reverse direction, perception during sliding is dominated by asperity collisions, whereas in the forward direction stickslip events dominate [33]; this is not inconsistent with mechanical instability arguments suggested earlier [12,32]. Note also that the friction coefficients themselves are largely a physical characterisation - no information is obtained for example related to sliding onset effects, which likely contribute to perception) $[34,35]$.

Further inspection suggested that the chemical composition of the surfaces, which could be split into two groups, 'lower' (1A, 1E and 1F) and 'higher' (1B, 1C and 1D) surface energy had resulted in variations during the curing process that could be attributed to surface tension. Namely, the lower surface energy surfaces tended to adopt a slight, tighter wrinkle pattern which somewhat increased the overall surface area of the coating, whilst the higher energy, more hydrophilic coatings tended to adopt a larger wavelength, slightly shallower wave pattern that-relative to the lower energy surfaces - tended to minimise surface area. Additionally, the relationship between the roughness and both the advancing and receding water contact angles was positive (where it would have been expected to be negative, since increasing the roughness of a hydrophilic surface is expected to decrease the water contact angle), suggesting that the differences in roughness were not contributing strongly to the measured water contact angles. The roughness of the surfaces in Series 1 was of relatively low amplitude (avg $\left.R_{\mathrm{p}}=2.01 \mu \mathrm{m}, \mathrm{sd}=1.22 \mu \mathrm{m}\right)$ and consisted of relatively smooth, shallow changes in amplitude (avg $R_{\mathrm{dq}}=1.55^{\circ}$, $\left.\mathrm{sd}=0.79^{\circ}\right)$. Given the positive relationship between the roughness $\left(R_{\mathrm{p}}\right)$ and the advancing and receding water contact angles, the contact angles may be presumed to mostly be related to the composition of the films rather than their topography, and may even be seen to describe the origin of the roughness, rather than being the result of it. This also explains the finding that whilst there was a weak correlation between differences in surface roughness and $d$ ', tactile friction was a much better fit (see Table 2). Not only was the roughness slight, but it was a direct result of the resin chemistry, and only served to enhance the differences in friction coefficient—-the more hydrophilic surfaces, which would be expected to have a higher tactile friction coefficient, were also smoother, whilst the slightly increased roughness of 
the less hydrophilic surfaces would be expected to further decrease their already lower tactile friction coefficient.

For Series 2, in which topcoat matting agents were varied, discrimination could be related to variation in roughness; specifically, the maximum peak height of the profile elements $\left(R_{\mathrm{p}}\right)$ was determined to be the strongest contributor to the discriminability of the stimulus pairs. This indicates that strategic manipulation of resins and matting agents to selectively influence the friction or roughness characteristics of a surface can indeed affect its haptic perception.

The physical parameters that were found to be relevant for discrimination in both Series 1 and 2 are consistent with key haptic dimensions reported in previous work. Series 1, where discrimination was related to the friction character, is reminiscent of the sticky-slippery dimension $[9,36]$. The results are in agreement with, for example, Skedung et al. [6] who argued that sticky-slippery is an important dimension of haptic touch and is directly associated with tactile friction. Series 2 seems instead to reflect the rough-smooth dimension of touch which has been discussed extensively [5, 9, 15, 37, 38].

According to the duplex theory of texture perception, perception of coarse features may be related to the slowly adapting receptor channels in the skin, whilst detection of features below $100-200 \mu \mathrm{m}$ is related to vibrations and in turn the Pacinian afferents in the skin [39]. Similarly, it has been suggested that coarse textural features may be represented as spatial patterns, whilst fine texture discrimination may be related to vibrations in the skin during movement [40]. Hollins and Risner [9] asked participants to discriminate between either two coarse (particle size $>100 \mu \mathrm{m}$ ) and two fine (particle size $<20 \mu \mathrm{m}$ ) abrasive papers in both a movement condition, where the paper was moved against their finger by the experimenter, and a static condition, where they placed their finger against the stationary stimuli. Although participants could discriminate the coarse papers in both conditions, they were unable to discriminate between the fine papers in the static condition. The authors concluded that because no vibration information was available in the absence of movement in the static condition, the ability to discriminate between the fine papers was heavily impeded. More recently, Skedung et al. [6] similarly showed that for pairs of surfaces of low amplitude, variations in the tactile friction coefficient were critical for differentiation by touch, and the results also indicated a role of topographic wavelength for the more textured surfaces. In both Series 1 and 2 here, the spacing of the features, characterised by $R_{\mathrm{sm}}$, was above $200 \mu \mathrm{m}$ and so discussing the results in terms of the duplex theory of texture perception may thus seem peculiar; but it is noteworthy that the most discriminable pair in the roughness series (Series 2) was $2 \mathrm{D}$ and $2 \mathrm{E}$. These had average feature spacings of $347 \mu \mathrm{m}$ and $261 \mu \mathrm{m}$, respectively, and the friction coefficient and average roughness were not remarkably different. (A figure portraying the line scans can be found in the ESI-Fig. S2) The value of $261 \mu \mathrm{m}$, however, is approaching the $200 \mu \mathrm{m}$ threshold often cited for vibration information to become more relevant for tactile perception. Bearing in mind that this is an average value it may well be that vibrational information can extend into this larger spacing range-particularly for surfaces which are not "purely particulate" as in the case of abrasive papers.

Ratings of pleasantness and naturalness of the surfaces were also affected by variations in the roughness and coefficient of friction. For pleasantness, it was found that surfaces with high friction and high roughness would likely be less preferred. These results are in agreement with the previous results suggesting that high friction surfaces were less preferred [24], but contrast with the results of Chen et al. [17] where it was found that sensations of stickiness positively contributed to perceived pleasantness. Klöcker et al. [24] suggested that the difference in results may be explained by differences in participants' finger moisture between their study and that of [17]. Finger hydration is known to affect tactile friction, for instance it was shown that higher finger moisture leads to higher friction because the elastic modulus of the stratum corneum is reduced, thus increasing the real area of contact at the asperity level [21]. However, as discussed by [41] finger moisture seems to increase tactile friction to a point, after which friction instead decreases with increasing moisture where liquid water modifies the contact. It is also possible that differences in the material properties of the stimuli selected could contribute to this discrepancy, as well as the fact that participants in [17] were given a specific context in which to assess the stimuli; namely that of use as confectionary packaging. Evidence from research investigating factors that can affect visual spatial evaluations suggests that subtle changes in the questions asked and the context in which tasks are embedded can lead to differences in the outcome of an experiment [42]. It is probably unreasonable to suppose that perceived pleasantness is independent of context. Providing the context of confectionary wrapping, as in [17] could induce associations of previous encounters with confectionary, which is useful within the parameters of that context but may be more difficult to generalise, although this speculation requires further testing. Nevertheless, these discrepancies highlight the fact that perceived pleasantness may be influenced by factors other than the material properties of the surface and the characteristics of the interaction with the surface. The emerging field of Psychotribology [12], namely the combination of tribology with psychophysics and psychology, will be integral in defining and addressing issues such as these in the future.

It is noteworthy that friction and roughness impact perceived naturalness in this study, given that the stimuli were not, in fact, natural at all (which is consistent with the systematically low naturalness scores). These results extend those of Overliet and Soto-Faraco [25] by demonstrating 
that the concept of perceived naturalness can be measured to some extent even in the absence of any stimuli that could be defined as natural. Despite this interesting and noteworthy outcome, the lack of truly 'natural' stimuli could also be seen as a limitation of the naturalness analysis here as it is possible that the inclusion of such stimuli would alter the provided naturalness ratings quite dramatically. For this reason, context is critical in the assessment of tactile perception. Perceived naturalness evidently has a more complex relationship to friction and roughness than does perceived similarity, but high roughness with (commensurately) low friction was, here, clearly perceived as most natural. For very low roughness surfaces, perceived naturalness seemed to be maintained with higher friction values, which may reflect previous findings that for smooth surfaces, participants have conflated high friction with roughness $[19,30]$ likely due to the vibrations induced by the stick-slip behaviour of the sliding contact between the finger and a more adhesive surface.

\section{Conclusions}

The manipulation of the tactile friction coefficient and topography by the variation of resins and matting agents in the topcoats of furniture finishes clearly allowed the identification of those parameters which manufacturers might choose to manipulate in order to tailor the haptic perception of their products. These relatively straightforward physical measurements could thus be used to specify coating quality from a haptic perspective-such specification is currently to all intents and purposes impossible.

Variation of the resins in otherwise identical furniture coating systems resulted in control of the tactile friction coefficient, which was responsible for the majority of the degree of differentiability of the surfaces. At the same time, these resin/friction changes strongly contributed to the perception of stickiness. By strategic control of the matting agents, it was possible to affect perceived smoothness, slipperiness and softness, which correlated with the roughness parameter known as the maximum profile peak height, $R_{\mathrm{p}}$. Because topography also necessarily affects friction, it was also found for the particulate matting agents that perceived stickiness and wetness correlated with the mean tactile friction coefficient.

Important perceptual dimensions in sustainable design are the perception of naturalness and pleasantness, and both these dimensions have here been clearly shown to be controllable via choice of resin and matting agent, and thus friction and topography. Perceived pleasantness was highest for those surfaces with low roughness and low friction and it was predicted that high roughness and high friction would be perceived as unpleasant. Perceived naturalness for these surfaces was associated with higher roughness and lower tactile friction coefficient. Increases in friction coefficient appeared to have a strong negative effect on perceived naturalness. For naturalness, the results suggest that without further context, surfaces with lower maximum peak height $\left(R_{\mathrm{p}}\right)$ feel only mildly natural regardless of the associated friction, whilst increasing friction rapidly decreases perceived naturalness on rougher surfaces.

It would appear that achieving surfaces that are perceived as both natural and pleasant in a furniture context represents a considerable challenge. Exquisite control of the haptic attributes can nonetheless be achieved using existing formulations and additives which allow friction and topography to become the palette for tactile aesthetics.

Supplementary Information The online version contains supplementary material available at https://doi.org/10.1007/s11249-021-01485-z.

Acknowledgements This work is an extract from a project predominantly financed by IKEA of Sweden AB and Akzo Nobel Coatings International B.V. We extend our thanks anonymously to all of the people there who contributed with practical assistance, materials choices and supported the concept.

Funding Open access funding provided by Royal Institute of Technology. This work is an extract from a project predominantly financed by IKEA of Sweden AB and Akzo Nobel Coatings International B.V.

\section{Declarations}

Conflict of interest The research was supported in part by funding provided by IKEA of Sweden AB and Akzo Nobel Coatings International B.V.

Ethical Approval The experiments involving humans presented in this manuscript were conducted in accordance with the Declaration of Helsinki (2013). When collecting and processing personal data, the principles of the General Data Protection Regulation (EU) 2016/679 (GDPR were adhered to. Informed consent was obtained in writing from all participants before the experiment began. No sensitive personal information was collected, and no invasive methods were used. The study was assessed for ethical compliance through an internal process at RISE and was approved by senior management at the Division of Surface, Process and Formulation.

Open Access This article is licensed under a Creative Commons Attribution 4.0 International License, which permits use, sharing, adaptation, distribution and reproduction in any medium or format, as long as you give appropriate credit to the original author(s) and the source, provide a link to the Creative Commons licence, and indicate if changes were made. The images or other third party material in this article are included in the article's Creative Commons licence, unless indicated otherwise in a credit line to the material. If material is not included in the article's Creative Commons licence and your intended use is not permitted by statutory regulation or exceeds the permitted use, you will need to obtain permission directly from the copyright holder. To view a copy of this licence, visit http://creativecommons.org/licenses/by/4.0/. 


\section{References}

1. Spence, C., Gallace, A.: Multisensory design: Reaching out to touch the consumer. Psychol. Mark. 28, 267-308 (2011)

2. Spence, C.: Tactile/Haptic Aspects of Multisensory Packaging Design. Multisensory Packaging, pp. 127-159. Springer, New York (2019)

3. Derler, S., Gerhardt, L.C.: Tribology of skin: Review and analysis of experimental results for the friction coefficient of human skin. Tribol. Lett. 45, 1-27 (2011)

4. Derler, S., Gerhardt, L.-C., Lenz, A., Bertaux, E., Hadad, M.: Friction of human skin against smooth and rough glass as a function of the contact pressure. Tribol. Int. 42, 1565-1574 (2009)

5. Bergmann Tiest, W.M., Kappers, A.M.: Haptic and visual perception of roughness. Acta Psychol. 124, 177-189 (2007)

6. Skedung, L., Arvidsson, M., Chung, J.Y., Stafford, C.M., Berglund, B., Rutland, M.W.: Feeling small: Exploring the tactile perception limits. Sci. Rep. 3, 2617 (2013)

7. Bensmaia, S.J., Hollins, M.: The vibrations of texture. Somatosens. Mot. Res. 20, 33-43 (2003)

8. Hollins, M., Bensmaïa, S., Karlof, K., Young, F.: Individual differences in perceptual space for tactile textures: Evidence from multidimensional scaling. Percept. Psychophys. 62, 1534-1544 (2000)

9. Hollins, M., Risner, S.R.: Evidence for the duplex theory of tactile texture perception. Percept. Psychophys. 62, 695-705 (2000)

10. Ndengue, J.D., Cesini, I., Faucheu, J., Chatelet, E., Zahouani, H., Delafosse, D., et al.: Tactile perception and friction-induced vibrations: discrimination of similarly patterned wood-like surfaces. IEEE Trans. Haptics 10, 409-417 (2016)

11. Carpenter, C.W., Dhong, C., Root, N.B., Rodriquez, D., Abdo, E.E., Skelil, K., et al.: Human ability to discriminate surface chemistry by touch. Mater. Horiz. 5, 70-77 (2018)

12. Skedung, L., Harris, K., Collier, E.S., Arvidsson, M., Wäckerlin, A., Haag, W., et al.: Feeling smooth: Psychotribological probing of molecular composition. Tribol. Lett. 66, 138 (2018)

13. Okamoto, S., Nagano, H., Yamada, Y.: Psychophysical dimensions of tactile perception of textures. IEEE Trans. Haptics 6, 81-93 (2013)

14. Kuroki, S., Sawayama, M., Sy, Nishida: Haptic metameric textures. BioRxiv (2019). https://doi.org/10.1101/653550

15. Bergmann Tiest, W.M., Kappers, A.M.: Analysis of haptic perception of materials by multidimensional scaling and physical measurements of roughness and compressibility. Acta Psychol. (Amst) 121, 1-20 (2006)

16. Skedung, L., Danerlöv, K., Olofsson, U., Johannesson, C.M., Aikala, M., Kettle, J., et al.: Tactile perception: Finger friction, surface roughness and perceived coarseness. Tribol. Int. 44, 505$512(2011)$

17. Chen, X., Shao, F., Barnes, C., Childs, T., Henson, B.: Exploring relationships between touch perception and surface physical properties. Int. J. Des. 3, 67-76 (2009)

18. Lederman, S.J., Taylor, M.M.: Fingertip force, surface geometry, and the perception of roughness by active touch. Percept. Psychophys. 12, 401-408 (1972)

19. Skedung, L., Harris, K.L., Collier, E.S., Rutland, M.W.: The finishing touches: The role of friction and roughness in haptic perception of surface coatings. Exp. Brain Res. (2020). https://doi. org/10.1007/s00221-020-05831-w

20. Zahouani, H., Mezghani, S., Vargiolu, R., Hoc, T., El Mansori, M.: Effect of roughness on vibration of human finger during a friction test. Wear 301, 343-352 (2013)

21. Arvidsson, M., Ringstad, L., Skedung, L., Duvefelt, K., Rutland, M.W.: Feeling fine-the effect of topography and friction on perceived roughness and slipperiness. Biotribology 11, 92-101 (2017)

22. Wenzel, R.N.: Surface roughness and contact angle. J. Phys. Chem. 53, 1466-1467 (1949)

23. Labbe, D., Pineau, N., Martin, N.: Food expected naturalness: Impact of visual, tactile and auditory packaging material properties and role of perceptual interactions. Food Qual. Prefer. 27, 170-178 (2013)

24. Klöcker, A., Wiertlewski, M., Théate, V., Hayward, V., Thonnard, J.-L.: Physical factors influencing pleasant touch during tactile exploration. PLoS ONE 8, e79085 (2013)

25. Overvliet, K.E., Soto-Faraco, S.: I can't believe this isn't wood! An investigation in the perception of naturalness. Acta Physiol. (Oxf) 136, 95-111 (2011)

26. Fujiwara, Y., Fujii, Y., Okumura, S.: Relationship between roughness parameters based on material ratio curve and tactile roughness for sanded surfaces of two hardwoods. J. Wood Sci. 51, 274-277 (2005)

27. Macmillan, N.A., Creelman, C.D.: Detection theory: A user's guide. Psychology press, London (2004)

28. Macmillan, N.A., Kaplan, H.L.: Detection theory analysis of group data: Estimating sensitivity from average hit and falsealarm rates. Psychol. Bull. 98, 185 (1985)

29. Tomlinson, S.E., Lewis, R., Carr, M.J.: The effect of normal force and roughness on friction in human finger contact. Wear 267, 1311-1318 (2009)

30. Arvidsson, M., Ringstad, L., Skedung, L., Duvefelt, K., Rutland, M.W.: Feeling fine-the effect of topography and friction on perceived roughness and slipperiness. Biotribology 11, 92-101 (2017)

31. Gao, L., McCarthy, T.J.: Contact angle hysteresis explained. Langmuir 22, 6234-6237 (2006)

32. Zhang, S., Rodriguez Urribarri, A., Morales Hurtado, M., Zeng, X., Van Der Heide, E.: The role of the sliding direction against a grooved channel texture on tool steel: An experimental study on tactile friction. Int. J. Solids Struct. 56, 53-61 (2015)

33. Zhou, X., Mo, J.L., Li, Y.Y., Xiang, Z.Y., Yang, D., Masen, M.A., et al.: Effect of finger sliding direction on tactile perception, friction and dynamics. Tribol. Lett. 68, 85 (2020)

34. Delhaye, B., Lefèvre, P., Thonnard, J.-L.: Dynamics of fingertip contact during the onset of tangential slip. J. R. Soc. Interface 11, 20140698 (2014)

35. Pasumarty, S.M., Johnson, S.A., Watson, S.A., Adams, M.J.: Friction of the human finger pad: Influence of moisture, occlusion and velocity. Tribol. Lett. 44, 117 (2011)

36. Yoshioka, T., Bensmaia, S.J., Craig, J.C., Hsiao, S.S.: Texture perception through direct and indirect touch: An analysis of perceptual space for tactile textures in two modes of exploration. Somatosens. Mot. Res. 24, 53-70 (2007)

37. Yoshida, M.: Dimensions of tactual impressions (1). Jap. Psychol. Res. 10, 123-137 (1968)

38. Ballesteros, S., Reales, J.M., de Leon, L.P., Garcia, B.: The perception of ecological textures by touch: does the perceptual space change under bimodal visual and haptic exploration? First Joint Eurohaptics Conference and Symposium on Haptic Interfaces for Virtual Environment and Teleoperator Systems World Haptics Conference, pp. 635-638. IEEE, New York (2005)

39. Hollins, M., Bensmaïa, S., Risner, R.: The duplex theory of tactile texture perception. In: Proceedings of the 14th annual meeting of the international society for psychophysics, pp. 115-121. The International Society for Psychophysics Québec, Canada (1998)

40. Weber, A.I., Saal, H.P., Lieber, J.D., Cheng, J.-W., Manfredi, L.R., Dammann, J.F., et al.: Spatial and temporal codes mediate the tactile perception of natural textures. Proc. Natl. Acad. Sci. USA 110, 17107-17112 (2013) 
41. Tomlinson, S., Lewis, R., Carré, M.: Review of the frictional properties of finger-object contact when gripping. Proc. Inst. Mech. Eng. J 221, 841-850 (2007)

42. Collier, E.S., Lawson, R.: Does grasping capacity influence object size estimates? It depends on the context. Atten. Percept. Psychophys. 79, 2117-2131 (2017)
Publisher's Note Springer Nature remains neutral with regard to jurisdictional claims in published maps and institutional affiliations. 